\title{
Characterization of amplification core and esterase B1 gene responsible for insecticide resistance in Culex
}

\author{
(gene amplification/organophosphate/detoxification/mosquitoes)
}

\begin{abstract}
Claude Mouches*†, Yves Pauplin $\ddagger$, Munna Agarwal*, Lynn Lemieux*, Michel Herzog§, Monique Abadon*, Valerie Beyssat-Arnaouty $\ddagger$, Olivier Hyrien ${ }^{*}$, Bruno Robert de Saint Vincent?, George P. Georghiou $\| * *$, AND Nicole Pasteur ${ }^{\ddagger}$
\end{abstract}

\begin{abstract}
*Unité de Génétique Moléculaire des Invertébrés, Institut National de la Recherche Agronomique, Station de Zoologie, P.0. Box 2078, 06606 Antibes, France; ‡Laboratoire de Génétique, Institut des Sciences de l'Evolution, Unité Recherche Associée 327, Centre National de la Recherche Scientifique, Université de Montpellier II, 34095 Montpellier, France; ${ }^{8}$ Département de Biologie Moléculaire et Cellulaire, Laboratoire Arago, Unité Associée 117, Centre National de la Recherche Scientifique, Université de Paris VI, 66650 Banyuls-sur-Mer, France; 'Laboratoire de Génetique Somatique, Institut Pasteur, 28 rue du Docteur Roux, 75724 Paris, France; and "Department of Entomology, University of California, Riverside, CA 92521
\end{abstract}

Communicated by Perry L. Adkisson, January 18, 1990

\begin{abstract}
Organophosphorus insecticide (OP) resistance in several Culex species is associated with increased esterase activity resulting from amplification of the corresponding structural gene. In Culex pipiens quinquefasciatus, high levels of $O P$ resistance $(\approx 800$ times) are due to the esterase B1 gene, which is amplified at least 250 -fold. This gene has now been sequenced, and the structure of the amplification unit (amplicon) encompassing the structural gene has been partially characterized. The inferred amino acid sequence of the enzyme revealed regions of strong homology with other eukaryotic serine-esterases, such as cholinesterases, which are the target of OPs. The amplicon covers at least 30 kilobases and contains a constant and highly conserved "core" of 25 kilobases. This core carries a single copy of the esterase gene ( 2.8 kilobases) as well as other sequences that are present as single or low number copies in the genomes of mosquitoes lacking overproduction of the esterase B1 protein. In the amplicon, the esterase gene is framed by two DNA sequences that are repeated in other parts of the genome of resistant mosquitoes and found in the genome of susceptible mosquitoes but not near the esterase B1 gene. It is suggested that these repetitive sequences may have a role in the amplification process.
\end{abstract}

Gene amplification seems to be a fundamental and widely occurring mechanism of overproduction of proteins for counteracting environmental stress. Amplification has been shown to result in high levels of resistance in insects that have been under selection with organophosphorus insecticides (OPs) (1-3), in alfalfa controlled with the herbicide Lphosphinothricin (4), and in Leishmania (5) and cultured mammalian cells (6) grown in the presence of antiproliferative drugs. Populations of several species of mosquitoes of the genus Culex have become resistant to a variety of OPs due to increased production of detoxifying esterases (7). The three esterases B studied thus far-i.e., B1, B2, and B3-were shown to be amplified $(1,2)$. There is evidence indicating that amplification of each esterase $B$ gene occurred as a single and independent event (2), suggesting that it may be induced by a specific mechanism. In order to explore the nature of this mechanism, we have undertaken the characterization of the amplified DNA structures. We report here the nucleotide sequence ${ }^{\dagger \dagger}$ and intron and exon organization of the esterase B1 gene, which, in Californian Culex pipiens quinquefasciatus, has been shown to be amplified at least 250 -fold (1). In addition, we show that large DNA sequences are coamplified

The publication costs of this article were defrayed in part by page charge payment. This article must therefore be hereby marked "advertisement" in accordance with 18 U.S.C. $\$ 1734$ solely to indicate this fact. with the structural gene and characterize part of these sequences.

\section{MATERIALS AND METHODS}

Strains. Mosquitoes used were as follows: $C$. p. quinquefasciatus strains S-Lab (susceptible), Tem-R [OP-resistant due to amplification of esterase B1 (1)], SeLax and Lahore [both containing an amplified esterase B2 (2)]; Culex pipiens pipiens strains S54 and MSE (OP-resistant due to esterase A1 and insensitive acetylcholinesterase, respectively); and Culex tarsalis strains S-Poso (susceptible) and Poso-Est [containing an amplified esterase B3 (2)].

Cloning Genomic DNA. Genomic DNA of Tem-R adults was partially digested by restriction enzyme Sau3AI. Sizeselected 15- to 22-kilobase (kb) DNA fragments thus generated were ligated to the BamHI site of the arms of phage vector $\lambda$-EMBL3. After packaging of the concatenated DNA molecules, the recombinant phage library was plated on Escherichia coli $\mathrm{C} 600$ at a density of 13,600 plaque-forming units per plate $(\phi=15 \mathrm{~cm})$. At this density, an average of 11 strongly positive signals per plate was obtained in the initial round of screening (8) using the $0.7-\mathrm{kb}$ cDNA probe coding esterase B1 (1). Seven recombinant phages (N1-N7) were purified in a first experiment and 28 (N8-N35) were purified in a second, through at least three cycles of plating and picking up of phage lysis, and were characterized by restriction mapping and blot hybridization. These recombinant phages were found to overlap, and the inserts in phages N5 and N7 (see Fig. 1) were found to extend the farthest on the $5^{\prime}$ and $3^{\prime}$ sides, respectively, of esterase B1 gene.

A second series of recombinants overlapping with the $5^{\prime}$ end of phage N7 or with the $3^{\prime}$ end of phage N5 were isolated from the genomic library by probes prepared from phages $\mathrm{N} 7$ and N5. Thus, on the $5^{\prime}$ end 29 recombinant phages (N400N428) were isolated with probe a $(1.15 \mathrm{~kb})$ prepared from phage N7 by Sal I-EcoRI digestion (Sal I being a site in the polylinker cloning sequence in EMBL3 vector), whereas on the $3^{\prime}$ end 27 recombinant phages (N2011-N2037) were purified using a $0.23-\mathrm{kb}$ probe prepared from phage $\mathrm{N} 5$ by $P v u$ I-Sal I digestion ( $\mathrm{Sal}$ I being a site in the polylinker cloning sequence in EMBL3 vector). Probe a and two of these recombinant phages, $\mathrm{N} 408$ containing a 14-kb insert and N2021 containing an 18-kb insert, are represented in Fig.

Abbreviation: OP, organophosphorus insecticide.

†Present address: Université de Pau, Département des Sciences Biologiques, Campus Universitaire, 64000 Pau, France.

**To whom reprint requests should be addressed.

${ }^{\dagger}$ The sequence reported in this paper has been deposited in the GenBank data base (accession no. M32328). 
1. Phages N5, N7, N408, and N2021 were used to establish a restriction map and to isolate various probes. Probe a prepared from phage N7 is described above. Probe $b$ is a 1.85-kb EcoRI fragment purified from phage N408. Probes c and $\mathrm{d}$ are $E$ coRI fragments of $8.0 \mathrm{~kb}$ and $2.6 \mathrm{~kb}$, respectively, obtained from phage N7. Probes e, $f$, and $g$ were isolated from phage $\mathrm{N} 5$; probe e is a 1.0-kb EcoRI fragment, probe $f$ is a 1.6-kb EcoRI-Bgl II fragment, and probe $\mathrm{g}$ is a $1.1-\mathrm{kb}$ EcoRI-Sal I fragment, the Sal I site of which comes from the EMBL3 vector. Probes $h$ and $i$ were isolated from phage N2021, probe h being a 0.6-kb $\mathrm{Xba}$ I-Cla I fragment and probe $\mathrm{i}$ being a 1.8-kb EcoRI fragment. Finally, probe $\mathrm{j}$ is a 1.5-kb EcoRI internal fragment of a repetitive element designated as "Juan." This Juan element was isolated from Tem-R genomic DNA library using probe g. Among the 13 phages studied, 10 contained the 1.5 -kb EcoRI fragment, and 1 of these phages (N13) was used to subclone fragment $j$.

Genomic DNA Studies. The 0.7-kb cDNA of esterase B1 and the phage DNA fragments (see above) were utilized as probes to study chromosomal DNA $(7.5 \mu \mathrm{g})$ digested to completion with restriction nucleases and subjected to electrophoresis on agarose gels $(0.8 \%)$. The separated fragments were transferred to nylon membranes and hybridized with ${ }^{32} \mathrm{P}$-labeled probes (specific activity, $2 \times 10^{6} \mathrm{cpm} / \mu \mathrm{g}$ ) in a buffer containing $0.2 \%$ SDS, $5 \times$ SSC $(1 \times$ SSC $=0.15 \mathrm{M}$ $\mathrm{NaCl} / 15 \mathrm{mM}$ sodium citrate), and $1 \times$ Denhardt's buffer $(0.02 \%$ bovine serum albumin/0.02\% Ficoll/0.02\% polyvinylpyrrolidone) during $16 \mathrm{hr}$ at $65^{\circ} \mathrm{C}$. The final washes were performed at $68^{\circ} \mathrm{C}$ in $0.2 \times \mathrm{SSC}$ and $0.2 \%$ SDS.

Esterase B1 Gene Structure. Genomic DNA from phage N5 and a 1.33-kb cDNA of esterase $B 1$ gene were sequenced after subcloning cDNA and phage insert fragments into M13mp18 and M13mp19, using the dideoxy chain-termination method according to Amersham recommendations. The 5 ' limit of the esterase $\mathrm{B} 1$ gene in phage $\mathrm{N} 5$ was identified by S1 nuclease mapping after hybridization with mRNA from Tem-R larvae and by partial sequencing. The 1.33-kb cDNA was the longest insert obtained by esterase $B 1$ immunological screening of the cDNA library that had been used to isolate the 0.7-kb cDNA mentioned above (1). It contains the whole sequence present in the $0.7-\mathrm{kb}$ cDNA. The $5^{\prime}$ end coding sequence of esterase $B 1$ was sequenced from mRNA by reverse transcriptase-mediated primer extension reaction on direct poly $(\mathrm{A})^{+}$template from Tem-R larvae. Three synthetic DNA oligomers complementary to the esterase B1 mRNA, corresponding to positions -2 to 25,942 to 965 , and 1253 to
1280 (see Fig. 3), were used successively to prime the sequencing reactions. Samples $(12.5 \mu \mathrm{l})$ of annealing buffer (100 mM KCl/50 mM Tris $\cdot \mathrm{HCl}, \mathrm{pH} \mathrm{8.3)} \mathrm{containing} 10 \mu \mathrm{g}$ of poly $(\mathrm{A})^{+}$mRNAs and $10 \mathrm{ng}$ of end-labeled primer were heated for $2 \mathrm{~min}$ at $90^{\circ} \mathrm{C}$ and allowed to cool at room temperature during $10 \mathrm{~min}$. The annealed mixture was placed on ice, and $5 \mu$ l of $5 \times \mathrm{RT}$ buffer $(250 \mathrm{mM}$ Tris $\cdot \mathrm{HCl}, \mathrm{pH}$ $8.3 / 250 \mathrm{mM} \mathrm{KCl} / 50 \mathrm{mM} \mathrm{MgCl}_{2} / 50 \mathrm{mM}$ dithiothreitol), $5 \mu \mathrm{l}$ of dNTP mixture $(2 \mathrm{mM})$, and $1 \mu$ l of avian myeloblastosis virus reverse transcriptase ( 25 units $/ \mu$ l) were added. Basespecific reactions were performed at $42^{\circ} \mathrm{C}$ for $30 \mathrm{~min}$ by mixing 4- $\mu$ l samples of this solution with $1 \mu$ l of one of the four ddNTP solutions (ddATP, $0.7 \mathrm{mM}$; ddCTP, $0.1 \mathrm{mM}$; ddGTP, $0.1 \mathrm{mM}$; ddTTP, $0.7 \mathrm{mM}$ ). A chase reaction was carried out for 15 min after adding $1 \mu$ of the dNTP mixture containing 1 unit of reverse transcriptase. Finally, $1 \mu$ l of RNase $(100 \mu \mathrm{g} / \mathrm{ml})$ was added and incubated for an additional $15 \mathrm{~min}$. Formamide-denatured aliquots of the base-specific reaction were analyzed by electrophoresis on $6 \%$ acrylamide/7 M urea gels.

\section{RESULTS}

Structure of the Amplification Unit. A restriction map of esterase $B 1$ gene and surrounding structures was established (Fig. 1) using ( $i$ ) the inserts isolated in phages N5 and N7 that hybridize with the $0.7-\mathrm{kb}$ cDNA of esterase B1 gene and (ii) the inserts isolated in phages N408 and N2021 that hybridize with the $5^{\prime}$ end of phage N7 and the $3^{\prime}$ end of phage N5, respectively.

The 35 recombinant phages (N1-N35) with an insert of Tem-R genomic DNA hybridizing with the $0.7-\mathrm{kb}$ esterase $\mathrm{B} 1$ cDNA contained a single (more or less complete) copy of the esterase B1 gene surrounded by DNA sequences that had identical restriction maps. In contrast, the recombinant phages hybridizing with probes isolated from the $5^{\prime}$ end of phage N7 and the $3^{\prime}$ end of phage N5 carried sequences that were identical on the "walking side" toward the esterase B1 gene but different at the opposite extremities. Thus, the cartography of the inserts present in the 29 phages isolated by probing with the $5^{\prime}$ end of phage $\mathrm{N} 7$ (probe a) contained the 1.85-kb EcoRI fragment $\mathrm{b}$ and other variable sequences, some of which hybridized with 2.6-kb EcoRI fragment d but had a different restriction map, and others with none of the fragments identified within phages N5 and N7. One such 1.2-kb EcoRI sequence was isolated from phage N408 and

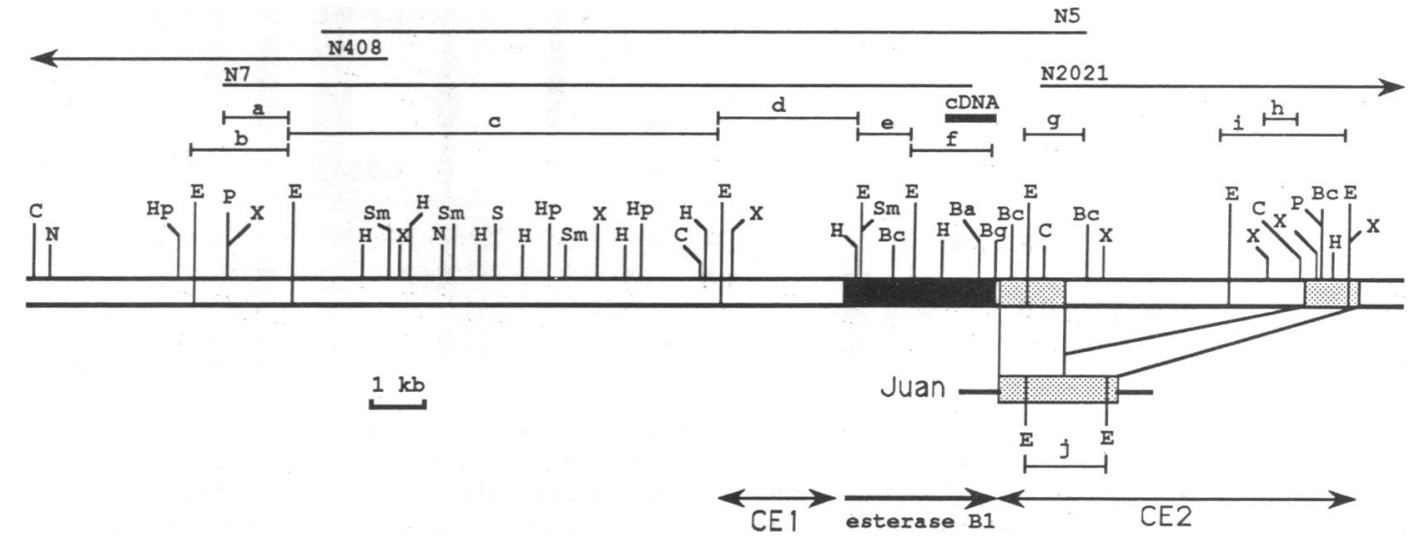

FIG. 1. Organization and maps of the constant region (core) of the amplicon containing esterase B1 gene obtained by endonuclease digestion of the various phage inserts. The long double line represents the genomic DNA core. Flanking sequences on either side of the core are heterogeneous. Each core carries a single copy of the esterase B1 gene (black box); the gene is framed by two elements, CE1 and CE2. The two ends of CE2 (stippled boxes) are parts of a highly repetitive element, Juan, represented below the amplicon. Above the amplicon are shown recombinant phages N5 and N7, part of recombinant phages $\mathrm{N} 408$ and N2021, as well as the 0.7-kb cDNA of esterase B1 and subcloned genomic fragments a to i. Ba, BamHI; Bc, Bcl I; Bg, Bgl II; C, Cla I; E, EcoRI; H, HindIII; Hp, Hpa I; N, Nco I; P, Pst I; S, Sal I; Sm, Sma I; X, $X b a$ I. Only some $B g l$ II and $B c l$ I sites are noted on the map. 
found to be amplified only 60 times in genomic Tem-R DNA that demonstrated a 250 -fold amplification when probed with the $0.7-\mathrm{kb}$ cDNA. Inserts of the 27 phages isolated by probing with the 3 ' end of phage N5 had little overlap with the N5 insert as exemplified by phage N2021 (Fig. 1). All of these phages contained the two EcoRI fragments $(4.0$ and $1.8 \mathrm{~kb}$, Fig. 1) as well as other EcoRI fragments that differed from each other at about $1 \mathrm{~kb}$ after the last $\mathrm{Xba}$ I site represented on the $3^{\prime}$ end of Fig. 1. One such EcoRI fragment $(1.5 \mathrm{~kb})$, isolated from phage $\mathrm{N} 2021$, disclosed an 80 -fold amplification in a slot blot experiment using genomic Tem-R DNA, which, when probed with the $0.7-\mathrm{kb}$ cDNA of esterase $\mathrm{B} 1$, disclosed a 250-fold amplification. Fig. 1 represents the regions that, based on the phage studies, show homogeneity and, therefore, strong conservation in the vicinity of esterase B1 gene.

The 0.7-kb cDNA and the subgenomic fragments a to $i$ (see Fig. 1) isolated from recombinant phages were used as probes for hybridizing blots of total genomic DNA of Tem-R insects that was digested by different restriction enzymes (Fig. 2). As expected from phage studies and restriction mapping, blot hybridizations using cDNA and fragments $a$ and $h$ revealed homogeneity in the sizes of the probed fragments when Tem-R DNA was digested by enzymes that cut within the conserved region but revealed heterogeneity when Tem-R DNA was digested by enzymes that do not cut within the probe or between the probe and the limit of the conserved sequence. Thus, a single, highly amplified band was observed (i) with cDNA probing after digestion by EcoRI, $\mathrm{HindIII,} \mathrm{Bcl}$ I, Cla I, and $X b a$ I (Fig. 2A, lanes 2-6), (ii) with fragment a probing after digestion by EcoRI (Fig. $2 B$, lane 2), and (iii) with fragment $h$ probing after digestion by $E c o R I, X b a I$, and HindIII (Fig. 2C, lanes 2-4). In contrast, several amplified bands were observed with fragment a probing after digestion with HindIII and $\mathrm{Bcl}$ I (Fig. $2 B$, lanes 3 and 4) or with fragment h probing after digestion with Sma I and BamHI (Fig. 2C, lanes 5 and 6).

Thus, our data indicate that in Tem- $R$ mosquito genome, esterase B1 genes are carried as single copies on a constant and highly conserved $25-\mathrm{kb}$ sequence representing the "core" of each amplification unit or amplicon. The core is the common part of the various amplification units that might have a much longer sequence. Each core contains seven EcoRI fragments of $1.85,8.0,2.6,1.0,2.1,4.0$, and $1.8 \mathrm{~kb}$ (Fig. 1). Amplification of most of these fragments was previously observed in Tem- $R$ mosquitoes by gel denaturation-renaturation of genomic DNA (9). The single copy of esterase $\mathrm{B} 1$ gene in each amplicon represents $<3 \mathrm{~kb}$ of the 25 $\mathrm{kb}$ of the core, extending mainly on the contiguous 1.0 - and 2.1-kb EcoRI fragments. The structure of the heterogeneous DNA sequences extending beyond the core probably results from rearrangements that have occurred during the amplification process. These DNA sequences include moderately amplified bands of various sizes, some of which hybridize with the 2.6-kb EcoRI fragment d present in the core of the amplicon (Fig. 2D, lane 3R). Some of these bands are not seen in the genome of susceptible insects (Fig. 2D, lane 3S), suggesting that they are specific to the amplicons.

As a consequence of their coamplification with the esterase B1 gene, all DNA sequences within the amplicon core are present in a large number of copies in Tem-R genomic DNA. In contrast, the number of copies of most of these sequences in the genome of OP-susceptible mosquitoes is considerably lower (Fig. 2D). Exceptions were two sequences in three EcoRI fragments of the amplicon core that are repeated in the genome of susceptible and OP-resistant mosquitoes. One such sequence, $\mathrm{CE} 1$, lies upstream of the esterase $\mathrm{B} 1$ gene within the 2.6-kb EcoRI fragment d, but its exact boundaries are not known. Other copies of this sequence are present in the heterogeneous $5^{\prime}$ region of the amplicon as discussed above. Use of the 2.6-kb EcoRI fragment $\mathrm{d}$ as a probe in blot hybridization experiments on EcoRI-restricted genomic DNA revealed the presence of reiterated sequences homologous to $\mathrm{CE} 1$ in the genome of $C$. p. quinquefasciatus and $C$. p. pipiens (Fig. $2 E$, lanes $1,3-5,7$, and 8 ) but not of $C$. tarsalis (Fig. 2E, lanes 2 and 6). The coamplification of the 2.6-kb EcoRI fragment d along with the esterase B1 gene drastically increases the abundance of this element in the genome of Tem-R insects (Fig. $2 E$, lane 1 ).
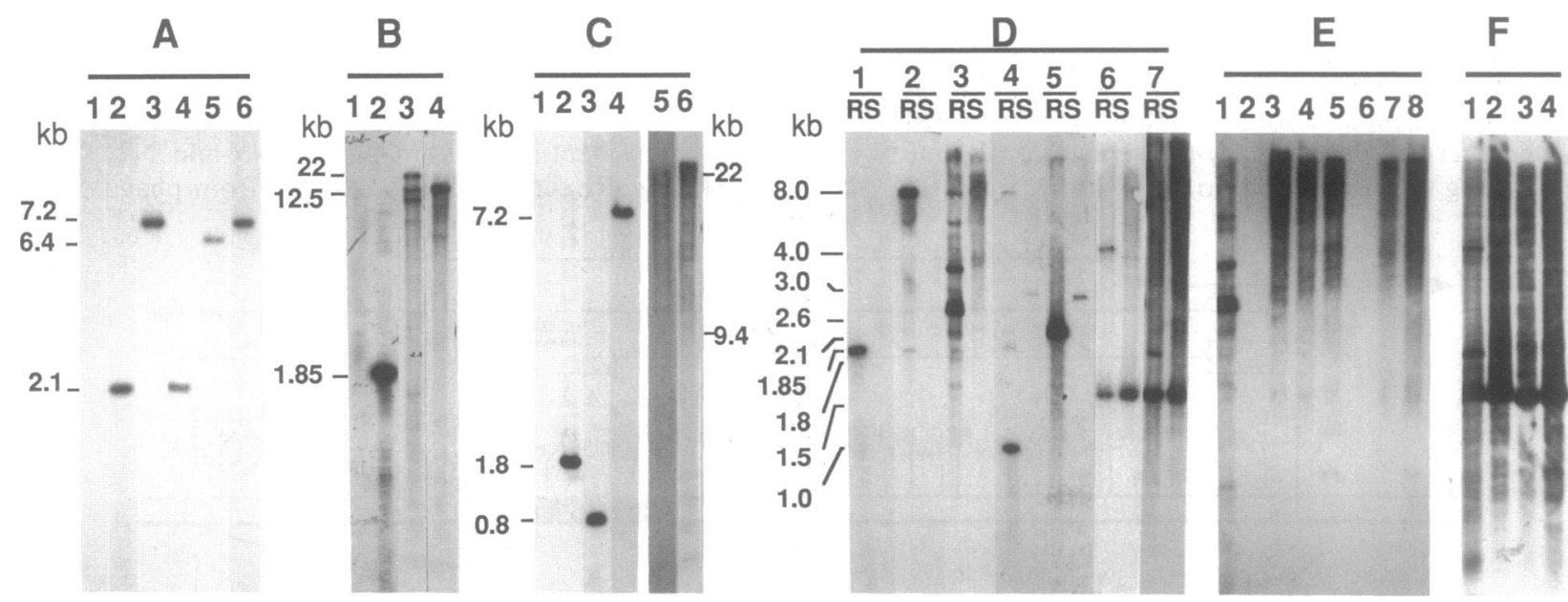

Fig. 2. Hybridization of genomic DNA from adult mosquitoes digested by various endonucleases with DNA probes specific for the esterase B1 amplicon and the Juan element. $(A)$ Use of esterase B1-specific 0.7-kb cDNA probe. Lanes: 1 and 2, EcoRI-digested genomic DNA from S-Lab and Tem-R mosquitoes, respectively; 3-6, DNA from Tem-R digested with HindIII, Bcl I, Cla I, and Xba I, respectively. (B) Use of genomic fragment a probe. Lanes: 1 and 2, EcoRI-digested DNA from S-Lab and Tem-R, respectively; 3 and 4, DNA from Tem-R digested with HindIII and Bcl I, respectively. (C) Use of genomic fragment $h$ probe. Lanes: 1 and 2, EcoRI-digested DNA from S-Lab and Tem-R, respectively; 3-6, DNA from Tem-R digested with Xba I, HindIII, Sma I, and BamHI, respectively. (D) EcoRI-digested genomic DNA of S-Lab (S) or Tem-R (R) mosquitoes probed with various fragments of the amplicon core. Lanes 1-7: fragments b to i, respectively. (E) EcoRI-digested genomic DNA of adult mosquitoes probed with 2.6-kb EcoRI fragment $d$ of the amplicon core. Lanes: $1,3,5$, and 8, C. p. quinquefasciatus strains Tem-R, S-Lab, SeLax, and Lahore, respectively; 4 and 7, C. p. pipiens strains S54 and MSE, respectively; 2 and 6, C. tarsalis strains S-Poso and Poso-Est, respectively. $(F)$ Hybridization of the 1.5-kb EcoRI internal fragment $\mathrm{j}$ of the Juan element with EcoRI-digested genomic DNA of adult mosquitoes. Lanes: 1 and 2,C.p. quinquefasciatus strains Tem-R and S-Lab, respectively; 3 and 4, C. p. pipiens strains MSE and S54, respectively. 
A second sequence repeated in the genome of resistant and susceptible mosquitoes corresponds to a major 1.5-kb EcoRI fragment that is detected by the use of probe $\mathrm{g}$ or $\mathrm{i}$ (Fig. 2D, lanes 6 and 7). This EcoRI 1.5-kb fragment is the central part of a highly repetitive $2.2-\mathrm{kb}$ DNA element designated as Juan (Fig. 1). Use of the 1.5-kb EcoRI fragment $\mathrm{j}$ of the Juan element as probe disclosed that Juan-like DNA represents about $3 \%$ of the total Culex DNA and seems to be scattered throughout the genome of every $C$. pipiens and $C$. tarsalis strain analyzed so far, regardless of insecticide resistance status (Fig. $2 F$ and unpublished data). In addition to a highly repeated $1.5-\mathrm{kb}$ band, fragment $\mathrm{j}$ detects several discrete bands on a smeared background (Fig. $2 F$ ). This might indicate that there exists a series of more or less internally deleted versions of this repetitive element. Alternatively, these bands could result from the accidental loss of one of the EcoRI sites; the frequent amplification of the new EcoRI band resulting from such mutations would indicate in turn a coamplification of these modified elements along with their flanking sequences. It will be important, therefore, to determine whether the presence of this repetitive element is associated with amplification of flanking sequences in other instances. In the core, the copy of Juan element has been split by the intercalation of a 4.4-kb sequence that is not highly repeated in the genome of susceptible Culex strains as revealed when probing genomic DNA with fragment h (Fig. $2 C$, lane 1). We designate this composite structure as CE2 (Fig. 1).

Structure of the Esterase B1 Gene. The complete 2.8-kb esterase gene structure was determined by sequencing a subgenomic DNA fragment of Tem-R mosquitoes cloned into phage N5 (Fig. 3). Exon areas were determined by sequencing a 1.33-kb cDNA encoding the esterase B1 carboxylic end. Determination of exons at the $5^{\prime}$ end of the gene was done by direct sequencing of esterase B1-specific mRNA. The es-

ACCGCAATAATCAAAATATAACAAMATAATGTTTTTITTCCTCTCAAATATGTTGAACAATCCCATTTTACCCCGCATCTGCATTCATTTTAAGAGAGA -301 ATTGTCTGTGTATAGGTAGAGTAGTCTCGCAGGTAGGGTACAAACACGAGCGATGAACACATTTGCATTTGCGATGAACACATTTGCTTGATGCGTGTTT -201 $>-. \rightarrow>-.->$

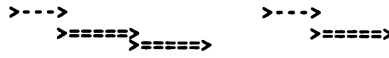

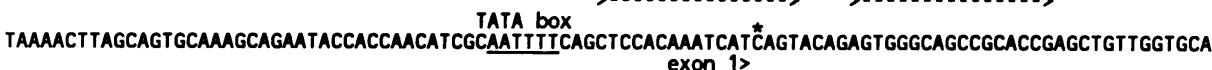
exon $1>$

AGTCAATTCAGCTGAGCAAACCGAAMAMAMAAACTTCGAAGAGTCACACCCAGCTGATAGCGAAAATTTAAGCAACAAAAAAACTCCAATCTACGTAGG

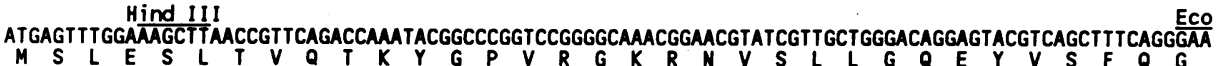

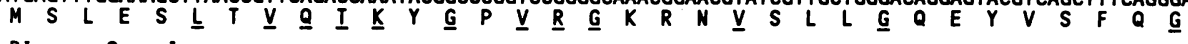
TTI TCGTACG Sma I $\underline{I} \underline{P} \underline{Y} R A \stackrel{P}{Q} \underline{G} \underline{L} \underline{R} \underline{E}$ <intron

TTGTTCAAATAAAGAGCAACGAGTGCTGCTGATTAGCGCTGTATCTAAGAGTGTGACCTCCGCGGCTTGATACTTCACCGCAATGACATGACATATTTGT IGATAAAAATAAAATGTAATAAATATTTTGTACTTGGACACGTACAAACCAGTCATGGTCTAGGCTGGAAATAAATTGAAGAGTGACAAAGTCAAACATA AATTTGGCAGTGACTTCCATTTTGATAMAGGTAAGACCATAAACTTAATCCAAAATTGTTTGTTGGACAATTGATAATGAAGTCTGTGCAAGTAGAAGT TATTGAAACATTAGACTGTTTAAAATTTAATTTMATCTATGATTTAATCTTTATTTTGTTCAATAATCTAAATTTTTGTAAAACAAAAAAATCTTCAGAA AACCTATCAACTGCATGCTATAACATTTTCAAATGACTTCGAAATATTACCGAMATACATTAMATTTGTCAATCAAATTTAGTAGTTTTGACATTTTGA

AAATGCTAAAAAAAT BCl I ATCACACCTCACTGTGTAAAATATTTTAACCAGCACTGTATTTCCCGGTGACTTGAACGCAAAACAAGCAACGAACAAAACTGGAAATTTAAAAACATAA

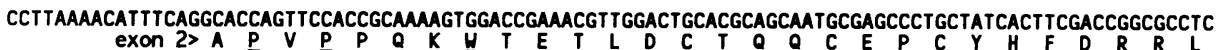

CAGAAGATCGTCGGCTGCGAGGACAGTCTGAAGATCAACGTGTTTGCGAAGGAGGTGAGTTGGTTTCAAAGAATCAATTTCAACTCTGAATTCACGATTT 110

CTCCAGATCAACCCTTCAACCCCTCTTCCGGTGATGCTGTACATCTACGGCGGGGCTTCACGGAAGGACCAGCGGAACCGAACTGTACGGGCCGGATT 1200

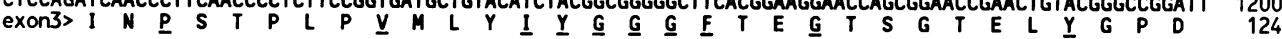
TCCTGGTTCAGAAGGATATCGTGTTGGTGTCGTTCAATTACCGTATTGGGGCGTTAGGTTTTCTGTGTTGTCAATCGGAGCAGGATGGCGTACCCGGTAA 1300 V $\quad \underline{S}$ F $N$ N TGCCGGACTCAAAGATCAGAACTTGGCCATTCGTTGGGTTCTGGAGACATTGCCGCCTTTGGAGgAGACCCGAAGCGCGTGACCCTGGCCGGCCATAGC 1400

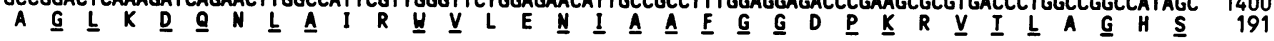
GCAGGTGCCGCTTCGGTTCAGTATCATCTGATTTCGGATGCGTCCAAGGACTTGTTTCAGCGGCGTATCGTAATGTCTGGGGTACGTATTCCAGTTGGT 1500

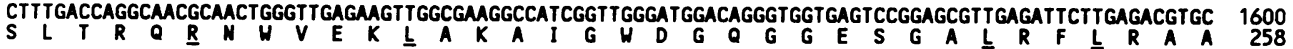
Hind 111

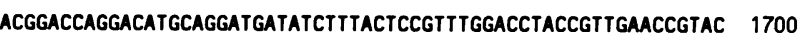

K P E D I V A H Q E K L L T D Q D M Q D D I F T P F G P T V E P Y 291 CTGACGGAACAGTGCATAATACCGAMGGCACCGTTCGAGATGGCTCGAACAGCTTGGGGTGCAAGATTGATATCATGATCGGTGGTACTTCTGAAGAAG 1800

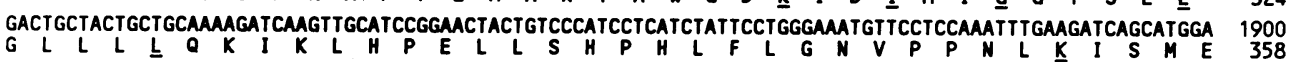
AAAACGAATCGAGTTTGCTGCCAAGCTGAACAACGTTACTACCCCGACAGCATTCCTTCAATGGAGAACAACCTGGGATACGTTCATGTAAGTCCAAAC 2000 K R I E F A A K L K Q R Y Y P D S I P S M E N N L G Y V H i intron 3 S CTAACCTCAATCACCACATCAACTTATCAACCCTCCACAMAATTCCAGATGATGTCCGACCGGGTCTTCTGGCACGGCCTGCACCGCACCATCCTTGCC 2100 CGCGCCGCTCGATCGCGCGCCCGCACCTTCGTGTACCGGATCTGTCTGGATTCGGAGTTTTACAACCACTACCGCATCATGATGATCGACCCGAAGCTGC 2200 $R A M A R S R$ A R T F V Y R I C L D S E F Y M H Y R I M M I D P K L T ${ }_{R}$ GCGGCACGGCCCATGCCGACGAGCTGTCCTATCTGTTTTCCAACTTTACCCAGCAGGTCCCCGGCAAGGAaACGTTCGAGTACCGCGGTCTGCAAACGCT 2300 GGTCGATGTGTTCAGCGCGTTCGTCATCAACGG Bam HI $V D V F S A$ E V I N G D P N C G M T A K G G V V E E P N A Q T K P 504 ACGTTCAAGTGTCTGAACATTGCCAACGACGGGGTGGCGTTCGTTGACTATCCGGATGCGGACCGGTTGGACATGTGGGACGCAATGTACGTGAATGATG 2500

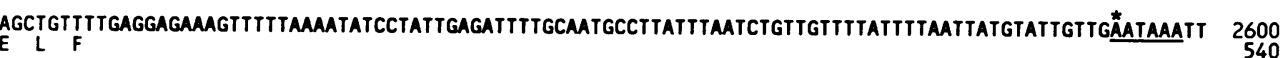

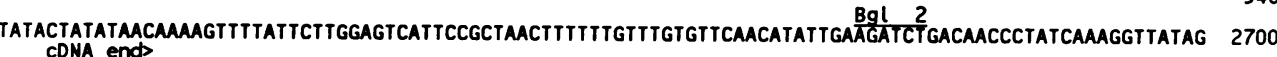

FIG. 3. Nucleotide sequence of esterase B1 gene and the inferred amino acid sequence derived from the exons' identified open reading frames. The major capping site and possible poly(A) site are marked by asterisks. The putative TATA box (AATTTT) and poly(A) signal are underlined. Arrows indicate direct repeats in the promoter region. Underlined amino acids are identical to those of the consensus sequence determined from human butyrylcholinesterase, Torpedo and Drosophila acetylcholinesterases, and Drosophila esterase 6 (10). 


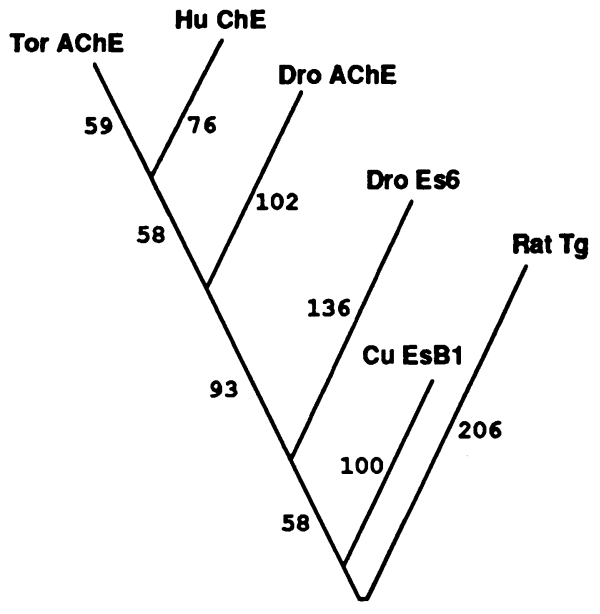

Fig. 4. Comparison of Culex esterase B1 (Cu EsB1) with the five most homologous proteins found in the Protein Identification Resource/National Biomedical Research Foundation database (release 18, October 1988, 9138 sequences). Tor AChE and Dro AChe, Torpedo and D. melanogaster acetylcholinesterases, respectively; Hu ChE, human butyrylcholinesterase; Dro Es6, D. melanogaster esterase 6; and Rat Tg, rat thyroglobulin. The phylogenetic tree, presented here with a tentative root, was constructed from sequence alignment of esterase B1 regions 6-25, 33-68, 99-147, 154-228, 231-243, 309-333, and 440-485. The topology of the tree was determined by the NJ method (17) using mutational distances between amino acids (18) and omitting gaps. The ancestral sequences were determined (19) and branch lengths were calculated as the minimum number of nucleotide substitutions between adjacent sequences (amino acid gaps having a weight of six nucleotide substitutions).

terase B1 gene is 2773 bp long and contains from the $5^{\prime}$ to $3^{\prime}$ end four exons of $279,138,882$, and $564 \mathrm{bp}$ and three introns of 775, 52, and $61 \mathrm{bp}$. Three direct repeats of 17,7 , and $5 \mathrm{bp}$ were identified, respectively, at positions -251 and -230 , positions $-240,-235$, and -220 , and positions $-258,-245$, and -224 , relative to initiation codon. No CCAAT box, in either orientation, was present upstream of the message, but it may be noted that the complementary sequence of one of the 3-fold repeats (i.e., CATTTG) is reminiscent of the canonical CCAAT box sequence. A putative TATA box (AATTTT) exists at position $-160,54 \mathrm{bp}$ after the last nucleotide of the direct repeats. Direct mRNA sequencing shows that one major capping site (position -138) is flanked on each side by three to five minor capping sites. A consensus poly(A) signal is present at the $3^{\prime}$ end of the gene, at position 2593. The inferred polypeptide encoded by the exons' open reading frames contains 540 residues (Fig. 3) and has a calculated $M_{\mathrm{r}}$ of 59,000 . Deduced amino acid sequence reveals three possible glycosylation sites (Asn-Xaa-Thr at amino acid 452, and Asn-Xaa-Ser at amino acids 20 and 95), and it presents from residues 189 to 196 the characteristics of the consensus 8-residue polypeptide shared at the active site of eukaryotic serine-esterases (11). In addition, esterase B1 contains regions strongly similar to human butyrylcholinesterase (12), acetylcholinesterases from Torpedo (13) and Drosophila melanogaster (14), and esterase 6 from Drosophila melanogaster (15). Substantial homologies were also observed with mammalian thyroglobulin (16). Regions of homologies with these proteins are found mainly between the $\mathrm{NH}_{2}$-terminal amino acid and the esterasic site (Fig. 3). The comparison of these regions (Fig. 4) indicates that esterase B1 is as different from Drosophila esterase 6 as this enzyme is from the three cholinesterases considered and thus probably belongs to a distinct subgroup.

\section{DISCUSSION}

Our results show that selection of Culex field populations by OP insecticides has resulted in amplification not only of the detoxifying esterase $\mathrm{B} 1$ gene but also of a large amount of DNA surrounding this gene. Part of this coamplified DNA is strongly homologous to middle or highly repetitive elements found in the genome of susceptible and OP-resistant insects. Although the nature of these elements is still unknown, the possibility that they are functionally related to transposable elements is of great importance in understanding the mechanism of gene amplification in insecticide-resistant insects.

We thank Chantal Quiles and Jo-Yanne Leberre (Institut National de la Recherche Agronomique-Antibes) for technical assistance. This research was supported by Institut National de la Recherche Agronomique (Grants 87.45.62 and 87.45.59) to C.M., Programme Interdisciplinaire de Recherches sur l'Environnement (Résistance aux Insecticides), Ministère de la Recherche et Enseignement Superieur (Grant 86-C-0593), Centre National de la Recherche Scientifique (Action Thematique Programmé Etats Unis), the National Science Foundation (INT-8715230) under the France-U.S. Collaborative Science Program, and the University of California Mosquito Research Program.

1. Mouchès, C., Pasteur, N., Bergé, J. B., Hyrien, O., Raymond, M., Robert de Saint Vincent, B., de Silvestri, M. \& Georghiou, G. P. (1986) Science 233, 778-780.

2. Raymond, M., Beyssat-Arnaouty, V., Sivasubramanian, N., Mouchès, C., Georghiou, G. P. \& Pasteur, N. (1989) Biochem. Genet. 27, 417-423.

3. Field, L. M., Devonshire, A. L. \& Forde, B. G. (1988) Biochem. J. 251, 309-312.

4. Donn, G., Tischer, E., Smith, J. A. \& Goodman, H. M. (1984) J. Mol. Appl. Genet. 2, 621-635.

5. Garvey, E. P. \& Santi, D. V. (1986) Science 233, 535-540.

6. Fukumoto, M., Shevrin, D. H. \& Roninson, I. B. (1988) Proc. Natl. Acad. Sci. USA 85, 6846-6850.

7. Mouchès, C., Magnin, M., Bergé, J. B., de Silvestri, M., Beyssat, V., Pasteur, N. \& Georghiou, G. P. (1987) Proc. Natl. Acad. Sci. USA 84, 2113-2116.

8. Benton, W. D. \& Davis, R. W. (1977) Science 196, 180-182.

9. Mouchès, C., Fournier, D., Raymond, M., Magnin, M., Bergé, J. B., Pasteur, N. \& Georghiou, G. P. (1985) C. R. Hebd. Acad. Sci. Sér. 3 301, 695-700.

10. Myers, M., Richmond, R. C. \& Oakeshott, J. G. (1988) Mol. Biol. Evol. 5, 113-119.

11. Dayhoff, M. O., ed. (1972) Atlas of Protein Sequence and Structure (Natl. Biomed. Res. Found., Washington, DC), Vol. 5.

12. Lockridge, O., Adkins, S. \& Ladu, B. N. (1987) J. Biol. Chem. 262, 12945-12952.

13. Schumacher, M., Camp, S., Maulet, Y., Newton, M., MacPhee-Quigley, K., Taylor, S. S., Friedmann, T. \& Taylor, P. (1986) Nature (London) 319, 407-409.

14. Hall, L. \& Spierer, P. (1986) EMBO J. 5, 2949-2954.

15. Oakeshott, J. G., Collet, C., Phillis, R. W., Nielsen, K. M., Russel, R. J., Chambers, G. K., Ross, V. \& Richmond, R. C. (1987) Proc. Natl. Acad. Sci. USA 84, 3359-3363.

16. Mercken, L., Simons, M.-J., Swillens, S., Massaer, M. \& Vassart, G. (1988) Nature (London) 316, 647-651.

17. Saitou, N. \& Nei, M. (1987) Mol. Biol. Evol. 4, 406-425.

18. Risler, J. L., Delorme, M. O., Delacroix, H. \& Henant, A. (1988) J. Mol. Biol. 204, 1019-1029.

19. Fitch, W. M. (1971) Syst. Zool. 20, 406-416. 